International Mathematical Forum, 1, 2006, no. 1, 41-46

\title{
Birationally very ample line bundles \\ on smooth curves
}

\author{
E. Ballico ${ }^{1}$ \\ Dept. of Mathematics, University of Trento \\ 38050 Povo (TN), Italy \\ ballico@science.unitn.it \\ C. Keem ${ }^{2}$ \\ Department of Mathematics \\ Seoul National University \\ Seoul 151-742, South Korea \\ ckeem@math.snu.ac.kr
}

\begin{abstract}
For all integers $r \geq 2$ and any smooth and connected projective curve $X$, let $\rho_{X}(r)$ denote the minimal integer $d$ such that there is a morphism $\phi: X \rightarrow \mathbf{P}^{r}$ birational onto its image and such that $\operatorname{deg}(\phi(X))=d$ and $\phi(X)$ spans $\mathbf{P}^{r}$. Fix integers $d, g$ such that $d \geq 8$ and $d^{2} / 6<g \leq d^{2} / 4-d$. Here we prove the existence of a smooth genus $g$ curve $X$ such that $\rho_{X}(3)=d$.
\end{abstract}

Mathematics Subject Classification: 14H50; 14H51

Keywords: Brill-Noether theory; space curve; quadric surface; Castenuovo's bound

\section{INTRODUCTION}

For all integers $r \geq 2$ and any smooth and connected projective curve $X$, let $\rho_{X}(r)$ denote the minimal integer $d$ such that there is a morphism $\phi: X \rightarrow \mathbf{P}^{r}$ birational onto its image and such that $\operatorname{deg}(\phi(X))=d$ and $\phi(X)$ spans $\mathbf{P}^{r}$. Suppose you have a morphism $\phi: X \rightarrow \mathbf{P}^{r}$ which is birational onto its image and whose associated base point free linear system is complete. Hence $\rho_{X}(r) \leq d$. It seems to be very hard to give conditions on $X$ which give $\rho_{X}(r)=d$. In [6] the authors considered the function $\rho_{X}(2)$. In particular, they give an existence theorem of smooth genus $g$ curves with prescribed $\rho_{X}(2)$ ([6], Prop. 2.2). We do not know if such an existence theorem (" no gaps for the integers $\rho_{X}(r)$ ") is true for some $r \geq 3$. Here we fill in an interval for the genus with prescribed $\rho_{X}(3)$ : when the genus is very large with respect to the integer $d:=\rho_{X}(3)$, near Castelnuovo's upper bound for space curves

\footnotetext{
${ }^{1}$ The author was partially supported by MIUR and GNSAGA of INdAM (Italy)

${ }^{2}$ Korea Research Foundation 2005-070-C00005
} 
with fixed degree ([5], Ch. III). To state our result we define the functions $\pi(d, 3)$ and $\pi_{1}(d, 3)$ as in [5], Ch. III.

For all integers $d \geq 7$, set $\pi(d, 3):=d^{2} / 4-d+1$ if $d$ is even, $\pi(d, 3):=\left(d^{2}-\right.$ 1) $/ 4-d+1$ if $d$ is odd, $\pi_{1}(d, 3)=d^{2} / 6-d / 2+1$ if $d \equiv 0(\bmod 3)$ and $\pi_{1}(d, 3)=$ $d^{2} / 6-d / 2+1 / 3$ if $d \equiv 1,2(\bmod 3)$.

Theorem 1. Fix integers $d, g$ such that $d \geq 8$ and $\pi_{1}(d-1,3)<g \leq \pi(d, 3)$. Then there exists a smooth genus $g$ curve $X$ such that $\rho_{X}(3)=d$.

Indeed, we will also find "large" families of curves genus $g$ curves with $\rho_{X}(3)=d$ (see Remarks 6 and 7).

We work over an algebraically closed field $\mathbb{K}$ with $\operatorname{char}(\mathbb{K})=0$.

\section{Proof of Theorem 1}

Example 1. Set $S:=\mathbf{P}^{1} \times \mathbf{P}^{1}$. Let $\pi_{1}: S \rightarrow \mathbf{P}^{1}$ and $\pi_{2}: S \rightarrow \mathbf{P}^{1}$ denote the two projections. We have $\operatorname{Pic}(S) \cong \mathbb{Z}^{\oplus 2}$ with, as generators, the class $\mathcal{O}_{S}(1,0)$ of a fiber of $\pi_{2}$ and he class $\mathcal{O}_{S}(0,1)$ of a fiber of $\pi_{1}$. We have $\omega_{S} \cong \mathcal{O}_{S}(-2,-2)$. We have $h^{0}\left(S, \mathcal{O}_{S}(a, b)\right)=0$ if either $a<0$ or $b<0, h^{0}\left(S, \mathcal{O}_{S}(a, b)\right)=(a+1)(b+1)$ if $a \geq 0$ and $b \geq 0, h^{1}\left(S, \mathcal{O}_{S}(a, b)\right)=0$, if either $a \leq-1$ and $b \leq-1$ or $a \geq-1$ and $b \geq-1, h^{1}\left(S, \mathcal{O}_{S}(a, b)\right)=(a+1)(-1-b)$ if $a \geq 0$ and $b \leq-2$ and $h^{1}\left(S, \mathcal{O}_{S}(a, b)\right)=$ $(-1-a)(b+1)$ if $a \leq-2$ and $b \geq 0$ (Künneth formula). For all integers $u \geq 0$ and $v \geq 0, \mathcal{O}_{S}(u, v)$ is spanned and $p_{a}(C)=u v-u-v+1$ for all $C \in\left|\mathcal{O}_{S}(u, v)\right|$. Set $\gamma(u, v):=u v-u-v+1$. For all $P \in S$ let $2 P$ denote the first infinitesimal neighborhood of $P$ in $S$, i.e. the closed subscheme of $S$ with $\mathcal{I}_{P, S}{ }^{2}$ as its ideal sheaf. Hence $(2 P)_{\text {red }}=\{P\}$, and length $(2 P)=3$. Fix any integral nodal curve $C \in\left|\mathcal{O}_{S}(u, v)\right|$ and set $A:=\operatorname{Sing}(C)$ and $a:=\sharp(A)$. Let $\nu: X \rightarrow C$ be the normalization map. Hence $p_{a}(X)=\gamma(u, v)-a$. Since $\omega_{S} \cong \mathcal{O}_{S}(-2,-2)$, we have $h^{0}\left(S, \omega_{S}\right)=h^{1}\left(S, \omega_{S}\right)=0$. Hence the classical adjunction theory developed for plane curves works for curves in $S$ and gives $H^{0}\left(X, \omega_{X}\right) \cong H^{0}\left(S, \mathcal{I}_{A}(u-2, v-2)\right)$.

Example 2. Let $T \subset \mathbf{P}^{3}$ be a quadric cone with vertex $P$ and $u: M \rightarrow T$ the blowing-up of $P$. Hence $M \cong F_{2}$ and $\operatorname{Pic}(M)$ is freely generated by a fiber $f$ of the ruling $\pi: M \rightarrow \mathbf{P}^{1}$ and by $h:=u^{-1}(P)$ (the section of $\pi$ with minimal selfintersection). We have $h^{2}=-2, h \cdot f=1, f^{2}=0$ and $\omega_{M} \cong \mathcal{O}_{M}(-2 h-4 f)$. Furthermore $\mathcal{O}_{M}(a h+b f)$ is spanned (resp. spanned and big, resp. very ample) if and only if $b \geq 2 a \geq 0$ (resp. $b \geq 2 a>0$, resp. $b>2 a>0$ ). The map $u$ is induced by the complete linear system $\left|\mathcal{O}_{M}(h+2 f)\right|$. Let $X$ be a smooth and projective curve and $\phi: X \rightarrow \mathbf{P}^{3}$ be a non-degenerate morphism which is birational onto its image and such that $\phi(X) \subset T$. Set $d:=\operatorname{deg}(\phi(X))$. Let $\mu$ be the multiplicity of $\phi(X)$ at $P$ and $C$ the strict transform of $\phi(X)$ in $M$. Then $d-\mu$ is even and $C \in\left|\mathcal{O}_{M}(((d-\mu) / 2) h+d f)\right|$. Hence $X$ has gonality at most $(d-\mu) / 2$. We have $\left.\omega_{C} \cong \mathcal{O}_{C}((d-\mu-4) / 2) h+(d-4) f\right)$ and hence $p_{a}(C)=1+\left(d^{2}-4 d-\mu^{2}\right) / 4$.

Remark 1. Take the set-up of Example 2. Fix an integer $u \geq 4$, an integer $u^{\prime} \geq u$, an integer $d>2 u^{\prime}$ and an integer $d^{\prime} \leq d-1$. Let $A \subset M$ be an integral curve, $A \in\left|u^{\prime} h+d^{\prime} f\right|$. We have $p_{a}(A)=1+u^{\prime}\left(d^{\prime}-u^{\prime}\right)-d^{\prime} \leq 1+u(d-1-u)-d+1<$ $1+u(d-u)-2 u=\gamma(u, d-u)$. Notice that $\gamma(u, d-u) \leq \gamma(x, d-x)$ for all $x$ such that $u \leq x \leq\lfloor d / 2\rfloor$. 
Remark 2. Let $N$ be a smooth and connected projective surface, $H$ an ample line bundle on $N$ and $F$ a vector bundle on $N$. For any torsion free sheaf $G$ on $N$ let $\mu(G, H):=\left(c_{1}(G) \cdot H\right) / \operatorname{rank}(G)$ denote its $H$-slope. Set $r:=\operatorname{rank}(F)$. We recall that $F$ is called $\mu$-semistable with respect to $H$ if $\mu(G, H) \leq \mu(F, H)$ for all nonzero subsheaves $G$ of $F$. Now assume that $F$ is $\mu$-semistable with respect to $H$. D. Gieseker proved in [3] the so-called Bogomolov-Gieseker's inequality: $c_{1}(F)^{2} \leq$ $(2 r /(r-1)) \cdot c_{2}(F)$. Fix a zero-dimensional subscheme $Z \subset N, Z \neq \emptyset$, and $L \in$ $\operatorname{Pic}(N)$. Let $E$ be any rank two vector bundle on $M$ fitting in an exact sequence

$$
0 \rightarrow \mathcal{O}_{N} \rightarrow E \rightarrow \mathcal{I}_{Z, N} \otimes L \rightarrow 0
$$

Assume that $L$ is nef and big. By [8], Lemma 2.3, (or see [1], Prop. 1.4, for a related result) $E$ is semistable, unless there is an effective divisor $D$ of $N$ such that $Z \subset D$ and

$$
L \cdot D-\operatorname{deg}(Z) \leq D^{2}<(L \cdot D) / 2<\operatorname{deg}(Z)
$$

Furthermore, if $(L \cdot D)^{2}=\left(L^{2}\right)\left(D^{2}\right)$ then $L$ and $\lambda D$ are linearly equivalent for some $\lambda \in \mathbb{Q}$ such that $2<\lambda \leq 1+\operatorname{deg}(Z) / D^{2}$ ([1], Prop. 1.4).

Lemma 1. Fix integers $u, v, a, b$ such that $v \geq u \geq 3,4 u>v, 0 \leq a \leq v-u$ and $v \geq u \geq c \geq 0$. Let $A \subset S$ be a general subset such that $\sharp(A)=a$. Then there is no $J \subset S$ such that $\sharp(J)=c, h^{1}\left(S, \mathcal{I}_{A \cup J}(u-2, v-2)\right) \neq 0$, and $\sharp\left(\pi_{2}(A \cup J)\right)=a+c$, except that if $v \leq u+1$ (and hence $a \leq 1$ ) we also require $\sharp\left(\pi_{1}(A \cup J)\right)=a+c$.

Proof. The assumption $\sharp\left(\pi_{2}(A \cup J)\right)=a+c$ implies $J \cap A=\emptyset$. The result is known and easy if $a=0$ (see below for the cases $1 \leq a \leq 3$ and [7] for our main application of Lemma 1 when $a=0$ ), Hence we will assume $a>0$ and that the result is true for the integer $a^{\prime}:=a-1$. For this fixed $a$ we may also assume $c>0$ and that the result is true for all $0 \leq c^{\prime}<c$. Assume the existence of $J \subset S$ such that $\sharp(J)=c$, $\sharp\left(\pi_{2}(A \cup J)\right)=a+c, \sharp\left(\pi_{1}(A \cup J)\right)=a+c$ and $h^{1}\left(S, \mathcal{I}_{A \cup J}(u-2, v-2)\right) \neq 0$. By our inductive assumptions we have $h^{1}\left(S, \mathcal{I}_{(A \cup J) \backslash\{P\}}(u-2, v-2)\right)=0$ for all $P \in A \cup J$, i.e $h^{0}\left(S, \mathcal{I}_{(A \cup J) \backslash\{P\}}(u-2, v-2)\right)=h^{0}\left(S, \mathcal{I}_{A \cup J}(u-2, v-2)\right)$ for all $P \in A \cup J$. Hence the set $A \cup J$ has the Cayley-Bacharach property with respect to the line bundle $\mathcal{O}_{S}(u, v)([4]$, p. 671 and p. 731, or [2] or [1]). Hence there is an exact sequence

$$
0 \rightarrow \mathcal{O}_{S} \rightarrow E \rightarrow \mathcal{I}_{A \cup J}(u, v) \rightarrow 0
$$

with $E$ locally free, $c_{1}(E)^{2}=2 u v$ and $c_{2}(E)=a+c$. Since $a+c \leq v$ and $u \geq 3$, we have $2 u v>4(a+c)$. Since $v \geq u>0$, the line bundle $L:=\mathcal{O}_{S}(u, v)$ is nef and big. By Remark 2 there is an effective divisor $D$ on $S$, say $D \in\left|\mathcal{O}_{S}(x, y)\right|$, such that $A \cup J \subset D$ and the inequalities (2) are satisfied, i.e.

$$
y u+x v-a-c \leq 2 x y<(y u+x v) / 2<a+c
$$

Since $A$ is general, we have $(x+1)(y+1) \geq a+1$. The inequalities

$$
y u+v x<2(a+c) \leq 2 v
$$

imply $x \leq 1$. We also have $2 x \leq u$ and $2 y \leq v$, because $L-2 D$ is effective ([1], 0.2). First assume $x=0$. Since $\sharp\left(\pi_{2}(A \cup J)\right)=a+c$, we have $y \geq a+c$. Hence from the first inequality in (5) we get $(a+c) u<v$. Hence $a+c \leq 3$ (here we use the assumption $4 u>v$ ). To complete this case we will do below (see parts (i), (ii) and (iii)) all cases with $a \leq 3$ assuming only the weaker inequality $y \geq a$. Now assume 
$x=1$. From (5) we get $y u<v$, while we also have $2 y \geq a-1$. Hence from (5) we get $(a-1) u<4 v$. Hence $a \leq 16$, because $4 u>v$. We take $y$ minimal and in this case we must distinguish the case " $D$ irreducible" (see part (iv) below) and the case " $D$ reducible" (see part (v) below).

(i) Assume $a=1$. From (4) we get $2+2 u>y u+x v$. Hence it is sufficient to check the following cases:

(i1) $y=0, x=1$;

(i2) $y=x=1, c=u, u \leq v \leq v+1$;

(i3) $y=0, x=2, v=u$.

In case (i1) $D$ is of type $(1,0)$, i.e. the set $A \cup J$ is contained in a fiber $F$ of $\pi_{2}$. Use the cohomology of $\mathcal{O}_{F}(u)$ and the surjectivity of the map $H^{0}\left(S, \mathcal{O}_{S}(u, v)\right) \rightarrow$ $H^{0}\left(F, \mathcal{O}_{F}(u)\right)$, true because $v \geq 0$. Similarly, in the case (i3) the set $A \cup J$ is contained in two fibers of $\pi_{2}$ and we conclude, just using that $v>0$. In case (i2) the set $A \cup J$ is contained in a curve $D \in \mathcal{O}_{S}(1,1)$. If $D$ is reducible, then we conclude as above using only that $u>0$ and $v>0$. If $D$ is irreducible, then $D \cong \mathbf{P}^{1}$ and we conclude because the restriction map $H^{0}\left(S, \mathcal{O}_{S}(u, v)\right) \rightarrow H^{0}\left(D, \mathcal{O}_{D}(u, v)\right)$ is surjective, $\operatorname{deg}\left(\mathcal{O}_{D}(u, v)\right)=u+v$ and $a+c \leq v \leq u+v+1$.

(ii) Assume $a=2$. From (4) we get $4+2 u>y u+x v$. Hence it is sufficient to check the following cases (here we use $v \geq u \geq 3$ ):

(ii1) $y=0,0 \leq x \leq 2, u \leq v \leq u+1$;

(ii2) $y=0, x=1$;

(ii3) $y=1, x=1, u \leq v \leq u+3$.

Case (ii2) is impossible, because two general points of $A$ have different images by the map $\pi_{2}$. Case (ii1) (resp. (ii3)) is done as case (i3) (resp. (i2)) of part (i).

(iii) Assume $a=3$. From (4) we get $6+2 u>y u+x v$. Furthermore, $(x+1)(y+$ $1) \geq 4$ and $v \geq u \geq 3$. Hence it is sufficient to check the following cases:

(iii1) $y=0, x=2, u \leq v \leq u+1$;

(iii2) $y=1, x=1, u \leq v \leq u+5$.

Case (iii) is done as cases (ii3). Case (iii2) is done as case (i2), distinguishing between the case " $D$ reducible" and the case " $D$ irreducible". First assume $D$ irreducible. Hence $D \cong \mathbf{P}^{1}$ and $\operatorname{deg}\left(\mathcal{O}_{D}(u, v)\right)=u+v+1$. Since $a+c \leq v \leq u+v+1$, the restriction map $H^{0}\left(D, \mathcal{O}_{D}(u, v)\right) \rightarrow H^{0}\left(A \cup J, \mathcal{O}_{A \cup J}(u, v)\right)$ is surjective. If $D$ is reducible, then a very weak form of our assumptions " $\sharp\left(\pi_{2}(A \cup J)\right)=a+c$ and $\sharp\left(\pi_{1}(A \cup J)\right)=a+c$ if $v \leq u+1$ " gives $a+c \leq 2$, contradiction.

(iv) Here we assume $x=1$ and $D$ irreducible. Since $2 y \leq v$ and $v \geq u \geq 4$ (the nefness of $L-2 D$ stated in [1], 0.2), we have $h^{1}\left(S, \mathcal{O}_{S}(u-3, v-y-2)\right)=0$. Hence the restriction map $H^{0}\left(S, \mathcal{O}_{S}(u-2, v)\right) \rightarrow H^{0}\left(S, \mathcal{O}_{D}(u-2, v-2)\right)$ is surjective. We get $h^{1}\left(S, \mathcal{I}_{A \cup J, S}(u-2, v-2)\right)=0$, contradiction.

(v) Here we assume $x=1$ and $D$ reducible. If $D$ is a union of a divisor of type $(1,0)$ and $y$ divisors of type $(0,1)$, then we have $y \geq \sharp\left(\pi_{2}(A \cup J)\right)=a+c$. Since $2 y \leq v$, this is a very easy case. Now assume that $D$ is the union of a divisor $D^{\prime}$ of type $\left(1, y^{\prime}\right), 0<y^{\prime}<y$, and $y-y^{\prime}$ divisors of type $y-y^{\prime}$. We easily get $A \subset A^{\prime}$. Set $J^{\prime}:=J \cap D^{\prime}$. By the minimality of $y$ we have $J^{\prime} \neq J$. By assumption (ii) and the minimality of $y$ we have $c-\sharp\left(J^{\prime}\right)=y-y^{\prime}$. Since $2 y \leq v$ and $v \geq u \geq 4$, we get again $h^{1}\left(S, \mathcal{O}_{S}(u-3, v-y-2)\right)=0$. Notice that $D^{\prime}$ contains exactly $a+c+y^{\prime}-y$ points of $A \cup J$, while each of the other components of $D$ contains exactly one point of $A \cup J$. 
Since $D^{\prime} \cong \mathbf{P}^{1}$, we get as in part (iv) that $h^{1}\left(S, \mathcal{O}_{(A \cup J) \cap D^{\prime}}\left(u-2, v-2-y+y^{\prime}\right)\right)=0$. Using $y-y^{\prime}$ Mayer-Vietoris exact sequences and that each component of $D \backslash D^{\prime}$ contains only one point of $(A \cup J) \backslash(A \cup J) \cap D^{\prime}$ we get $h^{1}\left(S, \mathcal{I}_{A, S}(u-2, v-2)\right)=0$, contradiction.

Remark 3. Fix integers $v \geq u \geq 3$ and $a$ such that $0 \leq a \leq v-u$. Let $A \subset S$ be a general subset with $\sharp(A)=a$. Set $Z:=\bigcup_{P \in A} 2 P$. It is very easy to check that $h^{1}\left(S, \mathcal{I}_{Z}(u, v)\right)=0, h^{0}\left(S, \mathcal{I}_{Z}(u, v)\right)=(u+1)(v+1)-3 a$ and that a general $C \in\left|\mathcal{I}_{Z}(u, v)\right|$ is integral, nodal and with $A=\operatorname{Sing}(C)$.

Remark 4. Let $C \subset S$ be an integral nodal curve and $\nu: X \rightarrow C$ its normalization. Set $A:=\operatorname{Sing}(C)$. Let $W$ be any base point free pencil on $X$. Fix any finite set $\left\{D_{i}\right\}_{i \in I}$ of integral curves on $S$, say $D_{i} \in\left|\mathcal{O}_{S}\left(x_{i}, y_{i}\right)\right|$, such that for every $i \in I$ the curve $D_{i}$ is the only element of $\left|\mathcal{O}_{S}\left(x_{i}, y_{i}\right)\right|$ containing the set $A \cap D_{i}$. Take a general $B \in W$. Since $W$ is base point free and $B$ is general in $W$ we have $A \cap B=\emptyset$ (i.e. $\sharp(\nu(B))=\sharp(B))$ and $\nu(B) \cap D_{i}=\emptyset$ for all $i \in I$. Now assume that any ramification point of $\left.\pi_{i} \mid(C \backslash A)\right): C \backslash A \rightarrow \mathbf{P}^{1}, i=1,2$, is an ordinary ramification point and that two such ramification points are mapped by $\pi_{i}$ onto different points of $\mathbf{P}^{1}$. This implies that there is no morphism $\alpha: X \rightarrow \mathbf{P}^{1}$, such that $\operatorname{deg}(\alpha) \geq 3$ and $\pi_{i} \circ \nu$ factors through $\alpha$. In this case either $W$ is induced by a ruling of $\pi_{i}$ or we have $\sharp\left(\pi_{i}(\nu(B))=\sharp(B)\right.$. Take the set-up of Remark 3. Our assumption on the ramification points of the map $\left.\pi_{i} \mid(C \backslash A)\right)$ is satisfied for a general $C \in \Gamma_{A}$.

Remark 5. Let $C \subset \mathbf{P}^{3}$ be an integral degree $d$ curve not contained in any quadric surface. Then $p_{a}(C) \leq \pi_{1}(d, 3)([5]$, Th. 3.13).

Remark 6. Take $C$ and $X$ as in Remarks 3 and 4. By Lemma $1 X$ has gonality exactly $u$. Hence the degree $u$ pencil $\left(\pi_{1} \mid C\right) \circ \nu$ is complete. Condition (iii) implies that for any non-constant morphism $f: X \rightarrow \mathbf{P}^{1}$ such that $f \neq\left(\pi_{1} \mid C\right) \circ \nu$, the morphism $\left(\left(\pi_{1} \mid C\right) \circ \nu, f\right): X \rightarrow S$ is birational onto its image and hence its image has arithmetic genus at least $u v-u-v+1-a$.

Remark 7. Take $v, u, a$ and $A$ as in Lemma 1 and set $\Gamma:=\bigcup_{A} \Gamma_{A}$, where the union is over all sufficiently general $A$. We get $\operatorname{dim}(\Gamma)=(u+1)(v+1)-a-1$. This wellknown equality could also be checked under far less restrictive assumptions on the integer $u, v, a$ just studying the normal sheaf of a nodal curve in $S$.

Proof of Theorem 1. By our assumptions on $d$ and $g$ there are integers $u, v$ such that $v \geq u \geq 3, u+v=d, 4 u>v$ and $\gamma(u-1, v+1)<g \leq \gamma(u, v)$. Set $a:=p_{a}(u, v)$ and fix a general $A \subset S$ such that $\sharp(A)=a$. Notice that $\gamma(u, v)-$ $\gamma(u-1, v+1)=v-u-1$. Hence $a \leq v-u$. By Remarks 3 and 4 we may apply Lemma 1. Hence the normalization, $X$, of any integral nodal curve $C \in\left|\mathcal{O}_{S}(u, v)\right|$ with $\operatorname{Sing}(C)=A$ has gonality $u$ and that any $g_{u}^{1}$ on $X$ is induced by a ruling of $S$. Hence there is no morphism $\psi: X \rightarrow S$ birational onto its image and with $\phi(X) \in\left|\mathcal{O}_{S}\left(u^{\prime}, v^{\prime}\right)\right|$ with either $u^{\prime}<u$ or $v^{\prime}<v$. Furthermore, since $p_{a}(\phi(X)) \geq$ $p_{a}(X)=g>\gamma(u, t)$, for all $t<v$ there is no such morphisms with $u^{\prime}=u$ and $v^{\prime}<v$. Hence there is no morphism $\phi: X \rightarrow \mathbf{P}^{3}$ birational onto its image, with $\operatorname{deg}(\phi(X))<d$ and with $\phi(X) \subset S$. By Remark 1 there is no morphism $\psi: X \rightarrow \mathbf{P}^{3}$ birational onto its image, with $\operatorname{deg}(\psi(X))<d$ and with $\psi(X)$ contained in a quadric cone. Since $g>\pi_{1}(d-1,3)$, we get $\rho_{X}(3)=d$. 


\section{REFERENCES}

[1] M. Beltrametti, P. Francia and A. J. Sommese, On Reider's method and higher order embeddings, Duke Math. J. 58 (1989), no. 2, 425-439.

[2] F. Catanese, Footnotes to a theorem of I. Reider, Algebraic Geometry, Proceedings, L'Aquila 1988, pp. 67-74, Lecture Notes in Mathematics 1417, Springer, Berlin, 1990.

[3] D. Gieseker, On a theorem of Bogomolov on Chern classes of stable bundles, Amer. J. Math. 101 (1979), no. 1, 79-85.

[4] P. Griffiths and J. Harris, Principles of Algebraic Surfaces, Wiley \& Sons, New York, 1978.

[5] J. Harris, with the collaboration of D. Eisenbud, Curves in Projective Space, Les Presses de l'Université de Montréal, Montréal, 1982.

[6] C. Keem and G. Martens, Curves without plane model of small degree, preprint.

[7] G. Martens, The gonality of curves on a Hirzebruch surface, Arch. Math. (Basel) 67 (1996), no. 4, 349-352.

[8] T. Nakashima, On Reider's method for surfaces in positive characteristic, J. Reine Angew. Math. 438 (1993), 175-185.

Received: August 31, 2005 